\title{
The Influence of Oral Malodor on Psychological Stress
}

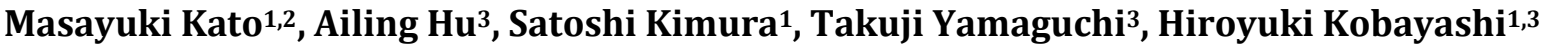 \\ ${ }^{1}$ Department of Hospital Administration, Graduate School of Medicine, Juntendo University, Tokyo, Japan \\ ${ }^{2}$ Ginza Daiko Dental Clinic, Tokyo, Japan \\ ${ }^{3}$ Center for Advanced Kampo Medicine and Clinical Research, Graduate School of Medicine, Juntendo University, Tokyo, Japan \\ Email: daiko677@amber.plala.or.jp
}

How to cite this paper: Kato, M., Hu, A., Kimura, S., Yamaguchi, T. and Kobayashi, H. (2019) The Influence of Oral Malodor on Psychological Stress. Health, 11, 501-510.

https://doi.org/10.4236/health.2019.115043

Received: February 7, 2019

Accepted: May 18, 2019

Published: May 21, 2019

Copyright (C) 2019 by author(s) and Scientific Research Publishing Inc. This work is licensed under the Creative Commons Attribution International License (CC BY 4.0).

http://creativecommons.org/licenses/by/4.0/

\begin{abstract}
We examined the influence of oral malodor on psychological stress among patients with a chief complaint of oral malodor. A total of 101 patients (40 male and 61 female) who visited our clinic with a chief complaint of oral malodor were included in this study. The primary compounds of oral malodor-volatile sulfur compounds (VSC) - were measured using the Oral Chro$\mathrm{ma}^{\mathrm{Tm}}$ instrument. Salivary stress markers, cortisol, and chromogranin A were measured using an enzyme-linked immunosorbent assay (ELISA) kit. The mood was measured using the Profile of Mood States 2nd Edition (POMS2). Among the participants, $60.3 \%$ had at least one level above the threshold in the following three markers: hydrogen sulfide, methyl mercaptan, and dimethyl sulfide. The gender distribution was 35 female (57.4\%) and 25 male (62.5\%). Salivary cortisol levels, a biomarker of stress, did not vary depending on the presence or absence of oral malodor. Chromogranin A levels were higher in the oral malodor female group than in the no oral malodor female group. With respect to the POMS2, regardless of the presence or absence of oral malodor measured using gas chromatography, the participants had higher scores for "Anger-Hostility" and "Fatigue-Inertia". Analysis by gender showed that "Depression-Dejection" was significantly higher in the oral malodor female group than in the no oral malodor female group. These results suggest that those who are worried about oral malodor have higher psychological stress than those who are not, regardless of the presence or absence of oral malodor measured using gas chromatography.
\end{abstract}

\section{Keywords}

Oral Malodor, Volatile Sulfur Compounds (VSC), Stress, Salivary Assay, POMS2 


\section{Introduction}

As the world becomes richer and more peaceful, increasingly more people are concerned about oral malodor and seeking cleanliness. However, in reality, most dental clinics have a difficult time determining how to deal with different types of patients with oral malodor. Although specialists rate the symptoms as mild, for patients, these are serious problems that can affect social acceptance. Hine M. K. pointed out that oral malodor causes psychological distress in many people who are worried about it [1]. Oral malodor is the generic name for objectively unpleasant odor from the oral cavity regardless of the cause. The symptom of "worrying that one's own body odor may cause distress to others" has been categorized as the olfactory reference syndrome, a type of social phobia, and has been studied in psychiatry. Since the 1980s, patients who worry excessively about their own oral malodor have been studied in the dental and psychosomatic fields. The symptom of "worrying that one's own body odor may cause distress to others," regardless of the presence or absence of oral malodor, has been categorized as self-perceived halitosis.

A 1999 survey on trends in health and welfare among 30,000 Japanese individuals conducted by the Japanese Ministry of Health, Labor and Welfare indicated public awareness of oral malodor in Japan. Seventy percent of those who have oral malodor have problems in the oral cavity. Indeed, $14.5 \%$ of them are worried about oral malodor [Reference]. Oral malodor is the fourth most common worry after periodontal diseases and dental caries. More than 10 million individuals in Japan are afraid of their oral malodor. A 1992 survey of oral malodor [2], as measured by volatile sulfur compounds (VSC) levels before lunch (i.e., when oral malodor is more likely to be experienced) among 2672 Japanese adults, showed that $23 \%$ of the respondents had significant oral malodor that can be socially problematic. As mentioned above, these results showed that a significant number of people have anxiety and complaints about oral malodor and that some of them do have oral malodor. However, it is another matter whether those who have anxiety or complaints about oral malodor actually have oral malodor. Another study found no significant relationship between having oral malodor and the self-recognition of it.

In younger generations, those who think they have oral malodor have significantly higher breath gas concentration than those who do not. On the other hand, in individuals over 45 years of age, those who think they have oral malodor have significantly lower breath gas concentration than those who do not.

Psychological factors that can directly affect interactions with others, such as worries about oral malodor, are subtle, and half of the patients with psychosomatic diseases have complaints of oral malodor. In terms of personality, those who are delicate perfectionists and those with mysophobia are likely to worry about oral malodor. It has been reported that experimentally induced psychological stress reduces the amount of saliva and increases oral malodor. The fact that oral malodor is mostly caused by psychological stress rather than by objec- 
tive factors shows that patients with complaints of oral malodor require effective and scientific psychological support.

There are few studies on the psychological stress of those who are worried about oral malodor. In this study, we examined the influence of oral malodor on psychological stress by using VSC levels, saliva stress markers, and a mood questionnaire.

\section{Materials and Methods}

\subsection{Participants}

Patients who visited Ginza Daiko Dental Clinic with a chief complaint of oral malodor were included in the study. A total of 101 patients (40 male and $61 \mathrm{fe}-$ male), after excluding those with systemic disease, Sjogren syndrome with a reduced amount of saliva, or severe periodontal disease, were included in this study. They provided informed consent prior to participation. This study was approved by the ethics committee of Medical Corporation Association Koyokai.

\subsection{Malodor Measurements (Breath Gas)}

Odor measurements and instrumental analysis were performed using a simple chromatography (Oral Chroma ${ }^{\text {tw }}$, FIS Corporation, Hyogo, Japan), which is less likely to be affected by temperature and humidity and allows high-precision measurements. The primary compounds of oral malodor, that is, VSC including hydrogen sulfide $\left(\mathrm{H}_{2} \mathrm{~S}\right)$, methyl mercaptan $\left(\mathrm{CH}_{3} \mathrm{SH}\right)$, and dimethyl sulfide $\left(\left(\mathrm{CH}_{3}\right)_{2} \mathrm{~S}\right)$, were measured in ppb for 8 minutes per measurement, using gas chromatography. The following thresholds were used: $\mathrm{H}_{2} \mathrm{~S}=112 \mathrm{ppb}, \mathrm{CH}_{3} \mathrm{SH}=$ $26 \mathrm{ppb}$, and $\left(\mathrm{CH}_{3}\right)_{2} \mathrm{~S}=8 \mathrm{ppb}$. Participants with levels exceeding at least one of the three thresholds were classified as those with oral malodor. All measurements were performed three hours after meals (i.e., between 10:00 am and 3:00 $\mathrm{pm})$.

\subsection{Measurements of Salivary Biomarkers}

Saliva collection was performed using the Saliva Collection Aid (SCA) (Salimetrics, LLC, State College, PA, USA). Specifically, saliva samples were collected by drooling using a straw that was inserted into a vial (a storage tube). The collected saliva was stored at -20 degrees until the analysis of salivary markers.

The levels of Salivary cortisol and chromogranin A in the collected saliva were measured using the ELISA assay and the Salivary Cortisol Enzyme Immunoassay Kit (Salimetrics, LLC, State College, PA, USA), respectively.

Chromogranin A levels were corrected with total protein measured using the BCA Protein Assay Kit (Pierce, Rockford, IL, USA).

\subsection{Mood Questionnaire}

The subjective mood was assessed using the Profile of Mood States 2nd Edition (POMS2), a 65-item questionnaire using a 5-point scale [3]. POMS2 is a ques- 
tionnaire that was developed to assess the subjective aspects of human emotions such as mood, feeling, and emotion. It measures seven mood scales: "Anger-Hostility", "Confusion-Bewilderment”, “Depression-Dejection”, "Fatigue-Inertia", "Tension-Anxiety", "Vigor-Activity" and "Friendliness". The implications of each scale are as follows:

1) “Anger-Hostility": a measure of hostility and anger toward others

2) "Confusion-Bewilderment": a measure of bewilderment and poor thinking ability

3) "Depression-Dejection": a measure of depression with loss of self-confidence

4) "Fatigue-Inertia": a measure of apathy and lack of vigor

5) "Tension-Anxiety": a measure of tension and anxiety

6) "Vigor-Activity": a measure of energy, uplifting feelings, and vigor

7) "Friendliness": a measure of sense of trust and compassionate and positive feelings toward others

The POMS2 was administered after saliva collection.

\section{Statistical Analyses}

All data are presented as mean \pm standard error. Statistical analysis was performed using the Wilcoxon signed rank test. P-values $<0.05$ were considered statistically significant.

\section{Results}

\subsection{Odor Concentration}

The results for the VSC (i.e., $\mathrm{H}_{2} \mathrm{~S}, \mathrm{CH}_{3} \mathrm{SH}$, and $\left(\mathrm{CH}_{3}\right)_{2} \mathrm{~S}$ ) are shown in Figure 1 . The measurements for $\mathrm{H}_{2} \mathrm{~S}, \mathrm{CH}_{3} \mathrm{SH}$, and $\left(\mathrm{CH}_{3}\right)_{2} \mathrm{~S}$ were $90.6 \pm 24.7$ (ppb), $24.1 \pm$ $7.6(\mathrm{ppb})$, and $19.6 \pm 5.2(\mathrm{ppb})$, respectively. Sixty-one participants (60.3\%) had at least one level above the threshold in the following three markers: hydrogen sulfide, methyl mercaptan, and dimethyl sulfide.

Forty participants (39.6\%) had levels below the threshold for all VSC markers. Based on these results, 61 participants were categorized as the oral malodor group. When analyzed for each VSC type, a high percentage of participants (34.7\%, 35 out of 61 participants) had only methyl sulfide levels above the threshold. Fourteen participants (13.9\%) had levels above the threshold for all three VSC.

Analysis by gender showed the incidence of oral malodor to be $62.5 \%$ and $57.4 \%$ in men $(\mathrm{n}=25)$ and women $(\mathrm{n}=35)$, respectively.

\subsection{Salivary Markers}

Measurements of salivary biomarkers, cortisol, and chromogranin A were performed. The cortisol and chromogranin A levels in the participants were $0.23 \pm$ $0.02 \mu \mathrm{g} / \mathrm{dl}$ and $9.94 \pm 0.73 \mathrm{pmol} / \mathrm{mg}$ protein, respectively. The results of the comparison between the oral malodor group and the no oral malodor group are shown in Figure 2. In the oral malodor group, the cortisol and chromogranin A 

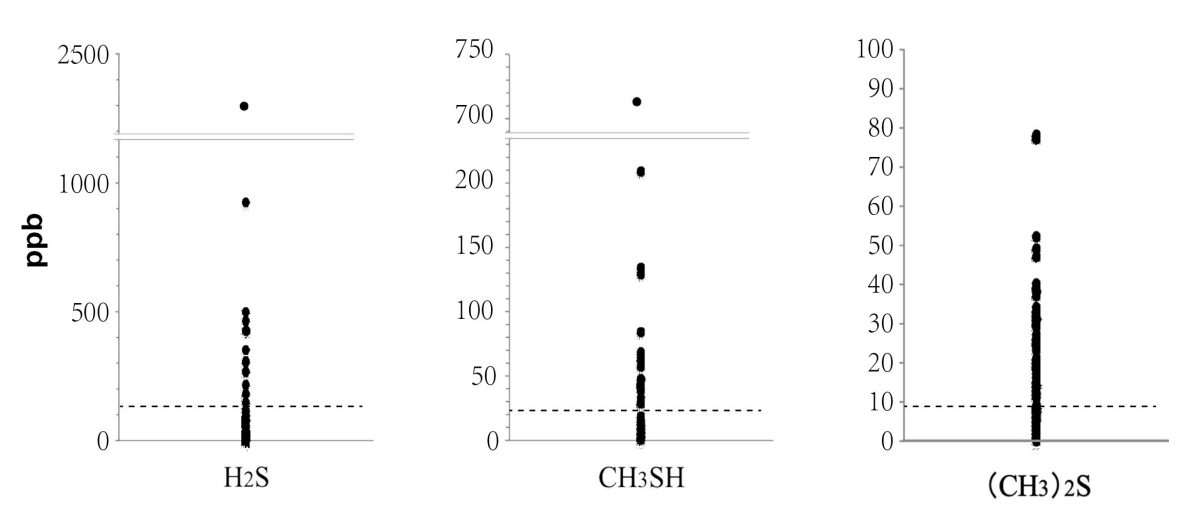

Figure 1. Measurements of volatile sulfur compounds. For the breath gas measurements, three volatile sulfur compounds (VSC) were measured using Oral Chroma ${ }^{\mathrm{Tm}}$ (FIS Corporation, Hyogo, Japan). The following thresholds for oral malodor were used: $\mathrm{H}_{2} \mathrm{~S}=112$ $\mathrm{ppb}, \mathrm{CH}_{3} \mathrm{SH}=26 \mathrm{ppb}$, and $\left(\mathrm{CH}_{3}\right)_{2} \mathrm{~S}=8 \mathrm{ppb}(\mathrm{N}=101)$.

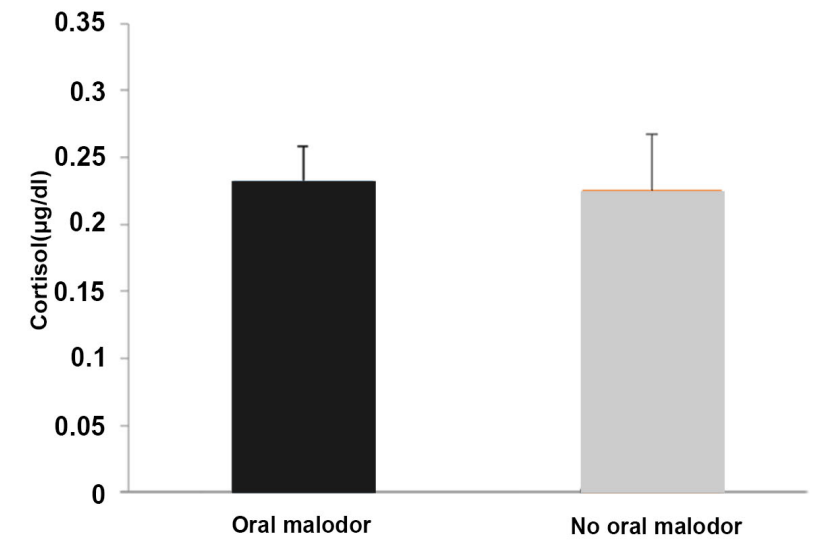

(a)

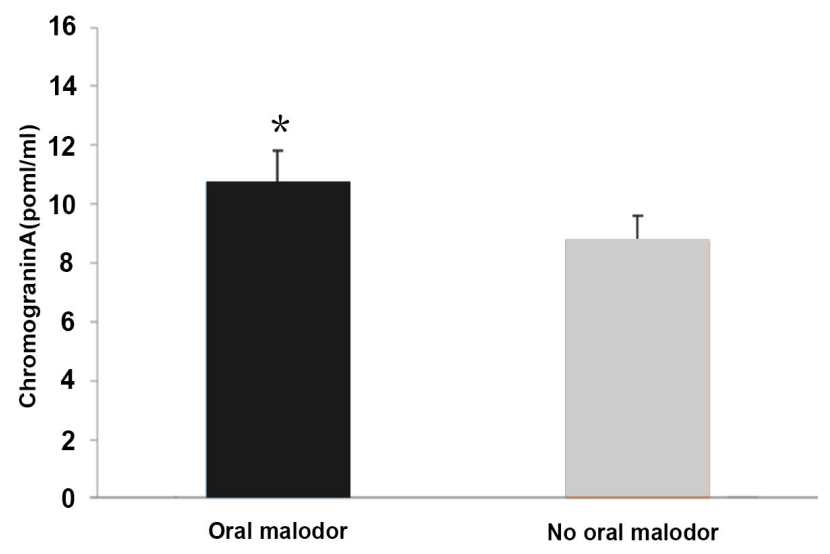

(b)

Figure 2. Salivary cortisol (A) and chromogranin A (B) levels in the participants (Mean \pm S.E., $N=101$ ), ${ }^{*}<0.05$ vs. No oral malodor.

levels were $0.23 \pm 0.03 \mu \mathrm{g} / \mathrm{dl}$ and $10.73 \pm 1.07 \mathrm{pmol} / \mathrm{mg}$ protein, respectively. In the no oral malodor group, the cortisol and chromogranin A levels were $0.23 \pm$ $0.04 \mu \mathrm{g} / \mathrm{dl}$ and $8.73 \pm 0.85 \mathrm{pmol} / \mathrm{mg}$ protein, respectively. The chromogranin A levels in the oral malodor group were significantly higher than those in the no oral malodor group.

The results of the analysis by gender are shown in Figure 3. For male participants, the cortisol and chromogranin A levels in the oral malodor group were $0.23 \pm 0.04 \mu \mathrm{g} / \mathrm{dl}$ and $8.41 \pm 0.92 \mathrm{pmol} / \mathrm{mg}$ protein, respectively. The cortisol and chromogranin A levels in the no oral malodor group were $0.22 \pm 0.04 \mu \mathrm{g} / \mathrm{dl}$ and $10.54 \pm 1.88 \mathrm{pmol} / \mathrm{mg}$ protein, respectively. For female participants, the cortisol and chromogranin A levels in the oral malodor group were $0.24 \pm 0.03 \mu \mathrm{g} / \mathrm{dl}$ and $12.45 \pm 1.68 \mathrm{pmol} / \mathrm{mg}$ protein, respectively. The cortisol and chromogranin A levels in the no oral malodor group were $0.23 \pm 0.04 \mu \mathrm{g} / \mathrm{dl}$ and $7.72 \pm 0.78$ $\mathrm{pmol} / \mathrm{mg}$ protein, respectively. Among male participants, the chromogranin A levels in the no oral malodor group were higher (but not significantly) than 


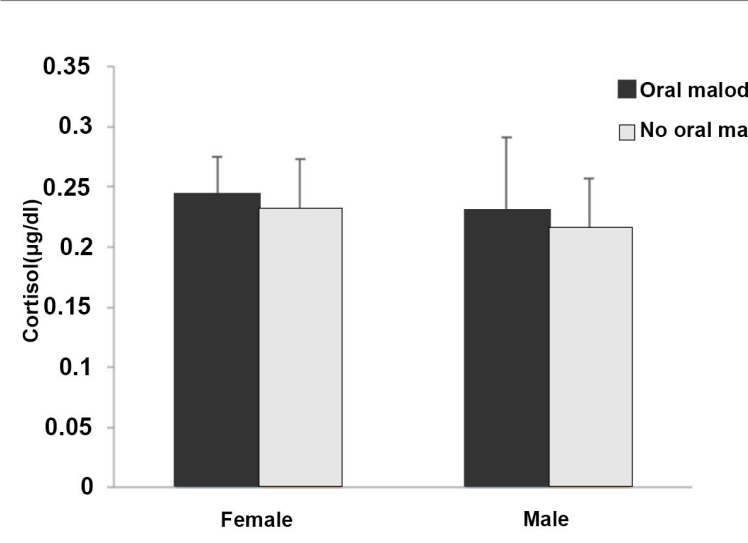

(a)

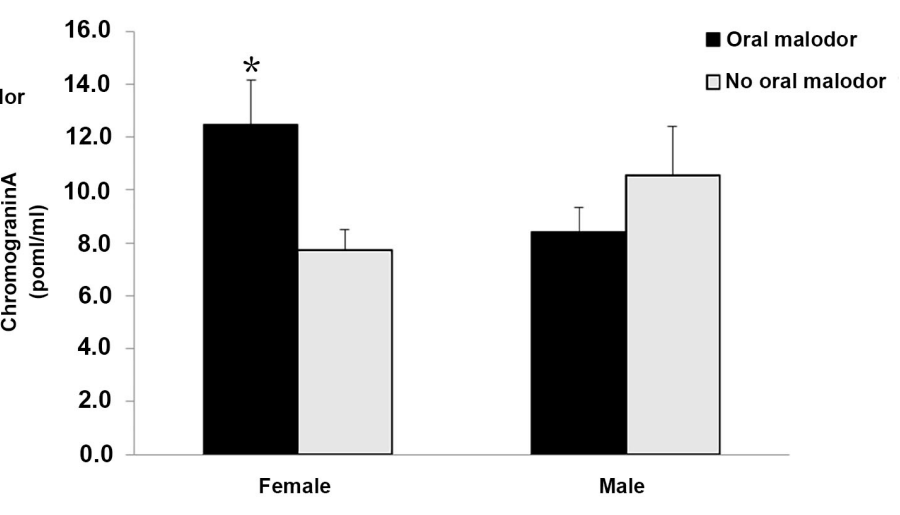

(b)

Figure 3. Salivary cortisol (A) and chromogranin A (B) levels by gender. Men (A, Mean \pm S.E. $n=40)$, Women (B, Mean \pm S.E. $n$ $=61),{ }^{\star}<0.05$ vs. No oral malodor.

those in the oral malodor group. Among female participants, the chromogranin A levels in the oral malodor group were higher than those in the no oral malodor group.

\subsection{Mood Questionnaire}

The participants' scores on the POMS2 are shown in Figure 4. Among the negative factors, Anger-Hostility ( $47.7 \pm 1.1$ in the no oral malodor group, $45.7 \pm 1.4$ in the oral malodor group) and Fatigue-Inertia (46.5 \pm 1.1 in the no oral malodor group, $45.4 \pm 1.2$ in the oral malodor group) were significantly higher in the no oral malodor group than in the oral malodor group. Among the positive factors, Friendliness ( $55.9 \pm 1.5$ in the no oral malodor group, $57.3 \pm 1.2$ in the oral malodor group) and Vigor-Activity (56.8 \pm 1.5 in the no oral malodor group, $55.5 \pm 1.2$ in the oral malodor group) showed differences (not statistically significant) between the two groups.

The results of the analysis by gender are shown in Figure 5 and Figure 6 . Among male participants, in the negative factors, Anger-Hostility (51.6 \pm 2.3 in the no oral malodor group, $45.8 \pm 2.5$ in the oral malodor group) and Depression-Dejection (50.2 \pm 1.3 in the no oral malodor group, $46.4 \pm 1.1$ in the oral malodor group) were significantly higher in the no oral malodor group than in the oral malodor group (Figure 5). Among female participants, in the negative factors, Depression-Dejection (47.4 \pm 1.1 in the no oral malodor group, $49.7 \pm$ 1.7 in the oral malodor group) was significantly higher in the oral malodor group than in the no oral malodor group.

Fatigue-Inertia ( $46.7 \pm 1.4$ in the no oral malodor group, $45.1 \pm 1.8$ in the oral malodor group was significantly higher) in the no oral malodor group than that in the oral malodor group (Figure 6). For both male and female participants, in the positive factors, Friendliness and Vigor-Activity in the oral malodor group were found to be significantly higher and lower than in the no oral malodor group, respectively. 


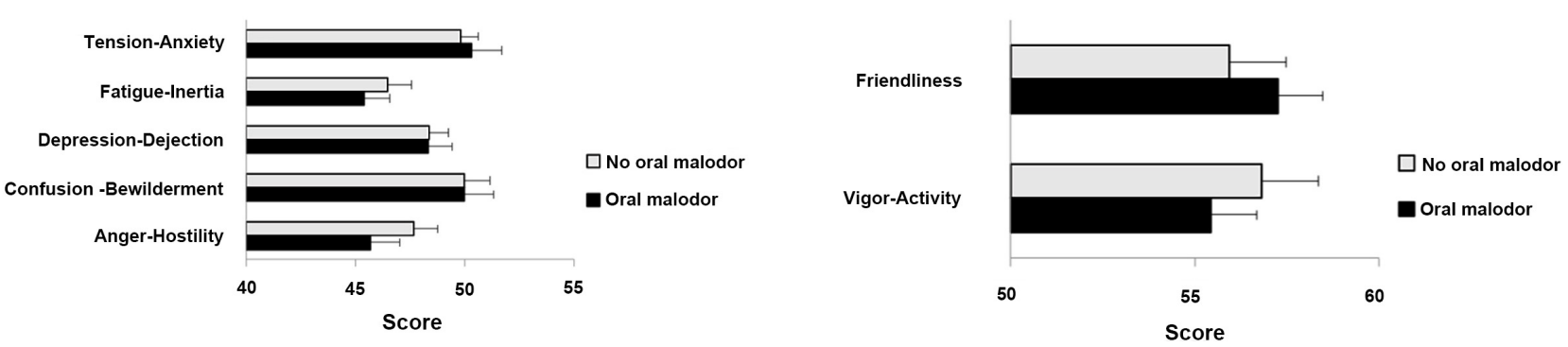

Figure 4. Scores on the profile of mood States 2nd edition (POMS2) in the participants (Mean \pm S.E., $\mathrm{N}=101$ ).
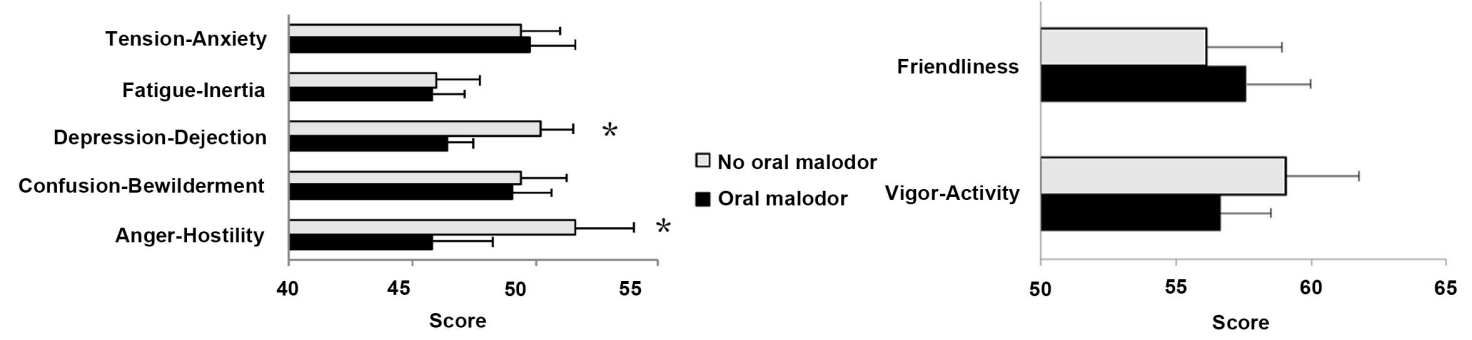

口No oral malodor

- Oral malodor

Figure 5. Scores on the Profile of Mood States 2nd Edition (POMS2) for male participants (Mean \pm S.E., $\mathrm{n}=40$ ), ${ }^{*}<0.05$ vs. Oral Malodor.
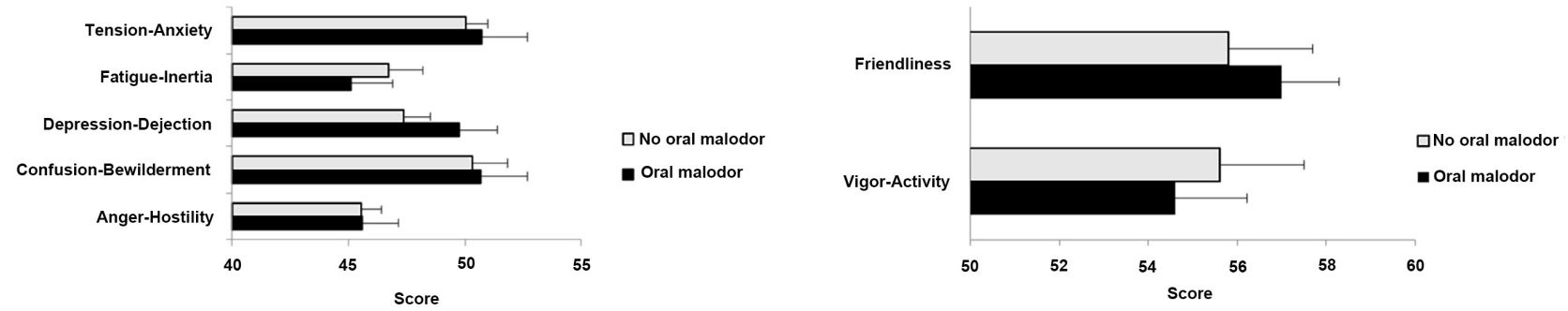

Figure 6. Scores on the Profile of Mood States 2nd Edition (POMS2) for female participants (Mean \pm S.E., $n=61$ ).

\section{Discussion}

In this study, we examined the influence of oral malodor on psychological stress by using gas chromatography, saliva stress markers, and a mood questionnaire in patients with a chief complaint of oral malodor.

Oral malodor was measured using three VSC markers that are the causative agents of oral malodor [4] [5]. These VSC markers have been associated with tongue coating, periodontal disease, and so on. The Oral Chroma ${ }^{\mathrm{Tm}}$ measurements showed that $60.3 \%$ of the participants had at least one level above the threshold of the following three markers: hydrogen sulfide, methyl mercaptan, and dimethyl sulfide. It is known that physiological oral malodor increases over time after a meal [5]. The results of this study are consistent with those by Nishioka et al. [6]. The results also suggest that oral malodor persists three hours after meals in two-thirds of healthy individuals.

We measured salivary stress markers, cortisol, and chromogranin A. Cortisol is an adrenocortical hormone that is secreted through the activation of the hypothalamic-pituitary-adrenal axis (HPA) system in response to various stressors 
[7] [8] [9]. Therefore, it is called a stress hormone. In this study, there was no significant difference in cortisol levels between the oral malodor group and the no oral malodor group. There were no significant gender differences in psychological stress between the two groups.

Chromogranin A is present in many secretory granules in endocrine organs including adrenal medullary chromaffin cells and sympathetic nerve endings. It responds to the action of the sympathetic nervous-adrenal medullary (SAM) system in the biological response to stress and is released into saliva by autonomic nerve stimulation [10] [11] [12] [13]. It is known that chromogranin A is useful as a psychological stress marker because it increases before cortisol levels begin to rise during psychological stress, decreases soon after the end of psychological stress, and responds poorly to physical stress. In this study, chromogranin A levels in the oral malodor group were significantly higher than those in the no oral malodor groups. Analysis by gender showed that chromogranin A levels in the female oral malodor group were significantly higher than those in the female no oral malodor group. These results suggest that, in women, oral malodor increases psychological stress. It has been reported that the amount of saliva decreases and oral malodor increases during psychological stress and that sympathetic and parasympathetic nerves are deeply involved in salivary secretion. The sympathetic nervous system is involved in the release of chromogranin A. Therefore, it may be activated by stress-induced oral malodor formation, reducing the amount of saliva. This study demonstrated that psychological stress increases oral malodor via the SAM system. It has been reported that autogenic training is effective in the treatment of oral malodor, suggesting that autonomic nerves are involved in the formation of oral malodor.

The analysis of the mood questionnaire showed that negative factors such as "Anger-Hostility" (a measure of anger and hostility towards others) and "Fatigue-Inertia" (a measure of apathy and lack of vigor) were significantly higher in the no oral malodor group than in the oral malodor group. The fact that participants without oral malodor, which was measured using gas chromatography, had higher scores for "Anger-Hostility" and "Fatigue-Inertia" suggests that they have difficulty interacting with others because of anxiety about oral malodor.

Analysis by gender showed that negative factors, such as "Anger-Hostility" and "Depression-Dejection" (a measure of depression with loss of self-confidence), were significantly higher in the male no oral malodor group than in the male oral malodor group. In the female participants, "Fatigue-Inertia" was significantly higher in the no oral malodor group than in the oral malodor group, while "Depression-Dejection" was significantly higher in the oral malodor group than in the no oral malodor group. For both male and female participants, among the positive factors, "Friendliness" (a measure of sense of trust and compassionate and positive feelings towards others) and "Vigor-Activity" (a measure of energy, uplifting feeling, and vigor) were found to be significantly higher and lower in the oral malodor group than in the no oral malodor group, respectively. 
The fact that the participants had higher scores for "Depression-Dejection" and lower scores for "Vigor-Activity" suggests that they have ambivalent feelings about wanting to get along with others and depression and anxiety about oral malodor.

These results suggest that those who are worried about oral malodor have higher psychological stress than those who are not, regardless of the presence or absence of oral malodor measured using gas chromatography. This study also showed that patients with oral malodor require not only dental treatment but also psychological treatment.

\section{Conflicts of Interest}

The authors declare no conflicts of interest regarding the publication of this paper.

\section{References}

[1] Hine, M.K. (1957) Halitosis. Journal of the American Dental Association, 55, 37-46. https://doi.org/10.14219/jada.archive.1957.0147

[2] Tonzetich, J. and Ng, S.K. (1976) Reduction of Malodor by Oral Cleansing Procedures. Oral Surgery, Oral Medicine, Oral Pathology, 42, 172-181. https://doi.org/10.1016/0030-4220(76)90121-3

[3] Heuchert, J.P. and McNair, D.M. (2012) Profile of Mood States 2nd Edition. Multi-Health Systems (MHS), Toronto. https://doi.org/10.1037/t05057-000

[4] Miyazaki, H., Sakao, S., Katoh, Y. and Takehara, T. (1995) Correlation between Volatile Sulphur Compounds and Certain Oral Health Measurements in the General Population. Journal of Periodontology, 66, 679-684. https://doi.org/10.1902/jop.1995.66.8.679

[5] Yaegaki, K. and Sanada, K. (1992) Volatile Sulfur Compounds in Mouth Air from Clinically Healthy Subjects and Patients with Periodontal Disease. Journal of Periodontal Research, 27, 233-238. https://doi.org/10.1111/j.1600-0765.1992.tb01673.x

[6] Nishioka, C., Hasegawa, N., et al. (2012) Halitosis in Daily Life: The Incidence and Factors Affecting the Onset of Symptoms. Journal of the Japanese Academy of Malodor Syndrome, 3, 7-11.

[7] Ohira, T., Okada, M. and Shimamoto, T. (2004) Effects of Foot Baths on Peripheral Skin Temperature, Blood Flow, and Salivary Cortisol. Biomedical Thermology, 23, 181-185.

[8] Kahn, J.P., Rubinow, D.R., Davis, C.L., Kling, M. and Post, R.M. (1988) Salivary Cortisol: A Practical Method for Evaluation of Adrenal Function. Biological Psychiatry, 23, 335-349. https://doi.org/10.1016/0006-3223(88)90284-3

[9] Hellhammer, D.H., Wüst, S. and Kudielka, B.M. (2009) Salivary Cortisol as a Biomarker in Stress Research. Psychoneuroendocrinology, 34, 163-171. https://doi.org/10.1016/j.psyneuen.2008.10.026

[10] Helle, K.B. (1966) Some Chemical and Physical Properties of the Soluble Protein Fraction of Bovine Adrenal Chromaffin Granules. Molecular Pharmacology, 2, 298-310.

[11] Winkler, H. and Fischer-Colbrie, R. (1992) The Chromogranins A and B: The First 25 Years and Future Perspectives. Neuroscience, 49, 497-528. 
https://doi.org/10.1016/0306-4522(92)90222-N

[12] Nakane, H., Asami, O., Yamada, Y. and Ohira, H. (2002) Effect of Negative Air Ions on Computer Operation, Anxiety and Salivary Chromogranin A-like Immunoreactivity. International Journal of Psychophysiology, 46, 85-89.

https://doi.org/10.1016/S0167-8760(02)00067-3

[13] Ng, V., Koh, D., Mok, B.Y., Chia, S.E. and Lim, L.P. (2003) Salivary Biomarkers Associated with Academic Assessment Stress among Dental Undergraduates. Journal of Dental Education, 67, 1091-1094. 